\title{
Marjolin's ulcer: A case report and literature review
}

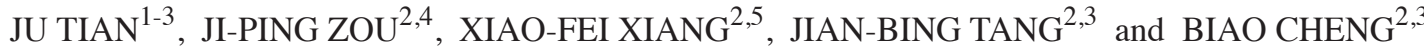 \\ ${ }^{1}$ Department of Plastic Surgery, Zhongshan City People's Hospital, Zhongshan, Guangdong 528400; \\ ${ }^{2}$ Department of Plastic Surgery, General Hospital of Southern Theater Command, PLA; \\ ${ }^{3}$ The Key Laboratory of Trauma Treatment and Tissue Repair of Tropical Area, PLA, \\ Guangzhou, Guangdong 510010; ${ }^{4}$ Guangzhou Medical Cosmetology Clinic, Guangzhou, \\ Guangdong 515041; ${ }^{5}$ Zhuhai Plastic Surgery Clinic, Zhuhai, Guangdong 519000, P.R. China
}

Received August 12, 2020; Accepted November 25, 2020

DOI: $10.3892 /$ wasj.2020.76

\begin{abstract}
There is currently no agreed standard for the definition, classification, etiology, pathological type, location, treatment and prognosis of Marjolin's ulcer (MU). The present study report a case of a 50-year-old male with inguinal lymph node and bone metastases from an MU on a burn scar on the left lower extremity. The patient carried this scar since he was 1 year old and had a chronic scar ulcer ever since. A histopathological examination of the inguinal lymph nodes and the ulcer demonstrated well-differentiated squamous cell carcinoma. Extensive resection was performed; however, the tumor eventually led to bone metastasis in the tibia. A more thorough second surgical resection that included the regional lymph nodes was more effective as the tumor did not recur. The present case report is complemented by presenting a review of the relevant literature, with an aim of aiding in the development of a preliminary clinical path for MU.
\end{abstract}

\section{Introduction}

Marjolin's ulcer (MU) is an ulcerating malignancy that occurs in chronically inflamed or scarred tissues (1). It can be classified into acute or chronic MU according to the latency period. No clearly defined cause for MU has been described. However, it is considered that the leading cause is mutations in focal cells due to chronic inflammatory. Based on the medical history, lesion biopsy and physical examination, MU can often be

Correspondence to: Professor Biao Cheng, Department of Plastic Surgery, General Hospital of Southern Theater Command, PLA, 111 Guangzhou Liuhua Road, Guangzhou, Guangdong 510010, P.R. China

E-mail: chengbiaocheng@163.com

Abbreviations: CT, computed tomography; MRI, magnetic resonance imaging; MU, Marjolin's ulcer; PET, positron emission tomography

Key words: Marjolin's ulcer, squamous cell carcinoma, chronic wound, burn scar, chronic inflammation accurately diagnosed. However, presently, there is no agreed standard for the definition, classification, etiology, pathological type, location, treatment and the prognosis of MU.

The present study reports a case of a 50-year-old male with inguinal lymph node and bone metastases from a MU in a burn scar on the left lower extremity. Furthermore, the relevant literature is reviewed, in the hope that this may aid the development of a preliminary clinical path for MU.

\section{Case report}

The present study was conducted in accordance with the 1975 Declaration of Helsinki. The study protocol was approved by the Ethics Committee of General Hospital of Southern Theater Command, People's Liberation Army. The patient provided written informed consent before investigations, screening, study and treatment.

A 50-year-old male presented with a 49-year history of a burn scar wound over the left lower extremity. When the patient was 1 year old, the skin of his left lower limb was burnt by fire. Following the change of the dressing, most of the wounds healed. However, a wound with a size of approximately $3 \times 2 \mathrm{~cm}$ behind the knee did not heal for a long period of time. In August 2011, the ulcer area had expanded, the wound edge had become red and swollen, and was accompanied by pain and apparent colorless exudation that occasionally turned red. Long-term antibiotic therapy and dressing change failed to heal the ulcer.

Following a dermatological examination, the patient was found to have an irregularly-shaped deep ulcer on the left lower extremity dorsum, which was approximately 36x16 cm. The wound granulation exhibited a cauliflower-like pattern, and a thick layer of necrotic material was observed. The edge of the ulcer was hard and everted, approximately $1 \mathrm{~cm}$ higher than the surrounding skin. A large amount of exudate was observed on the wound, and a foul odor was present. The left inguinal lymph nodes were enlarged. The size of the lower lymph node group was approximately $3 \times 5 \mathrm{~cm}$, and the upper group consisted of 5-6 lymph nodes of approximately $1 \mathrm{x} 1 \mathrm{~cm}$. The enlarged lymph nodes were somewhat hard, with no apparent tenderness, a low degree of activity, and no adhesion to the surrounding tissues. No bone tissue damage was detected on leg radiographs (Fig. 1). A biopsy of the ulcerative 
A

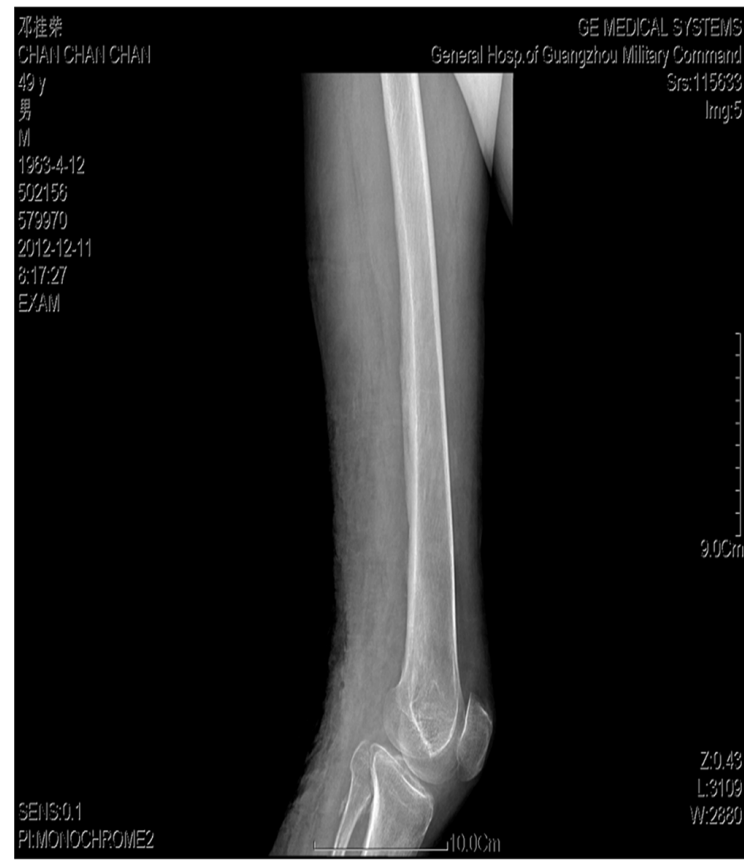

B

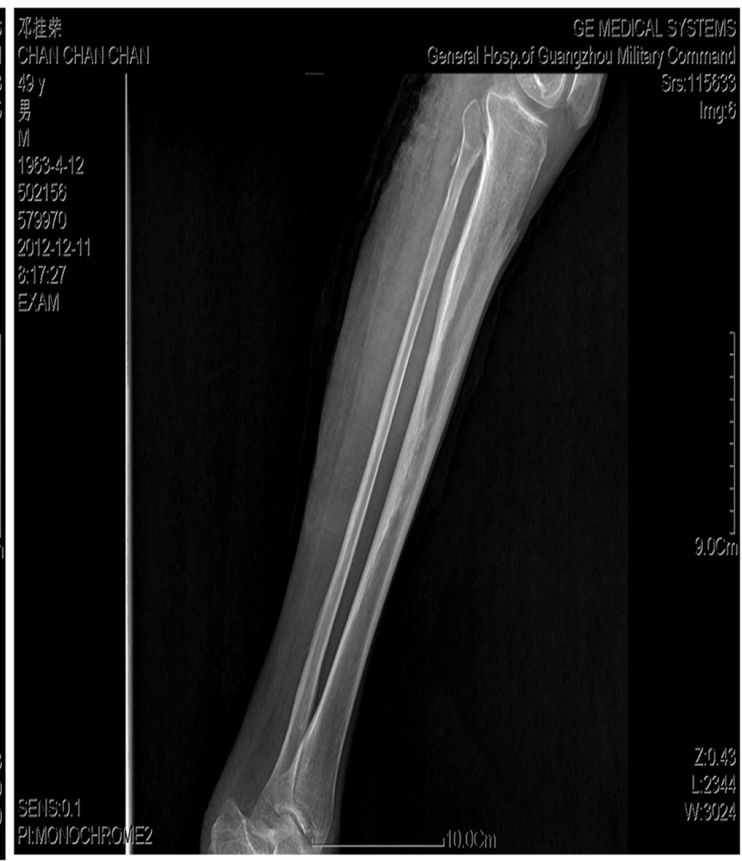

Figure 1. Radiographs of the affected leg. No bone tissue damage could be detected on these images. (A) Left femur; (B) Left tibia and fibula.

A

B

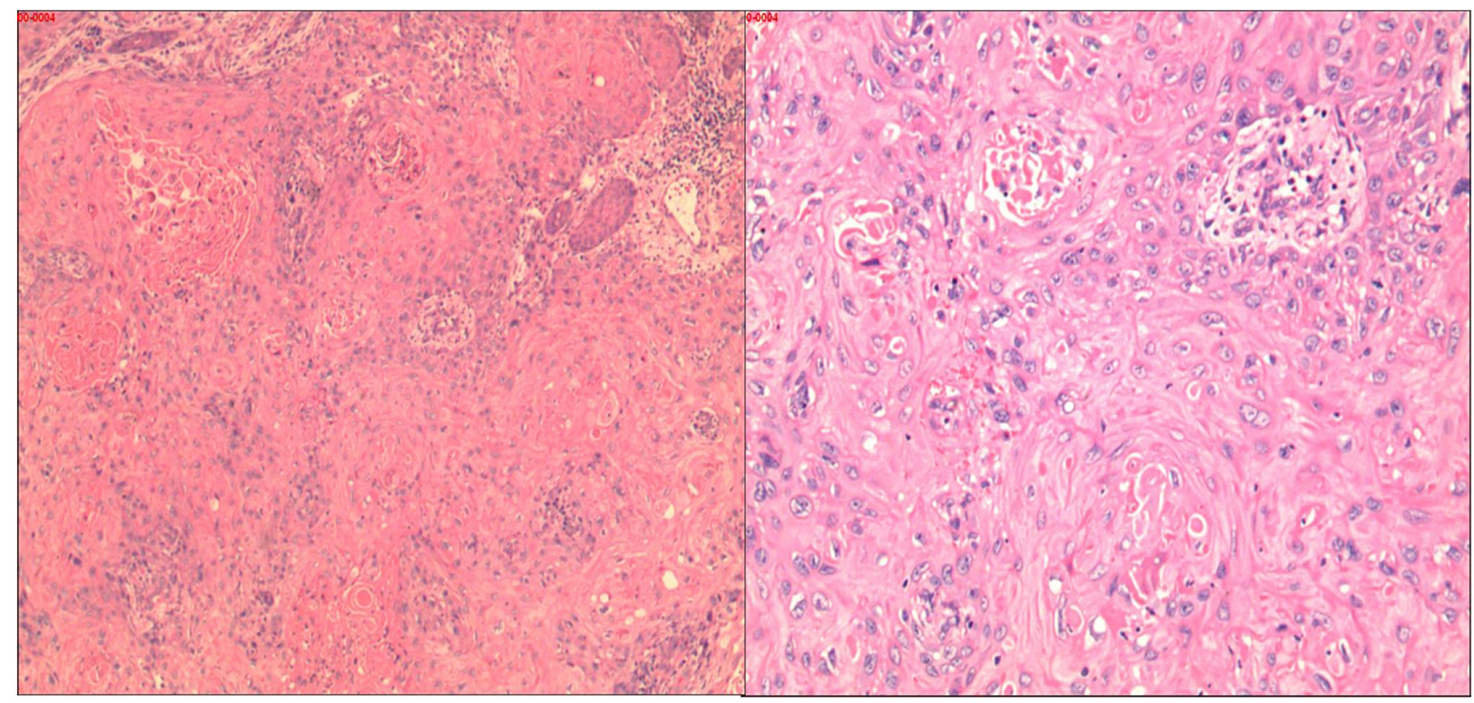

Figure 2. Histopathology of the ulcer tissue. Highly differentiated squamous cell carcinoma can be observed. Hematoxylin and eosin staining at a magnification of (A) x100 and (B) x200.

tissue (performed by a pathologist) revealed highly differentiated squamous cell carcinoma (Fig. 2).

The patient refused to undergo computed tomography (CT) or magnetic resonance imaging (MRI) in search of metastases from the lesion and objected to lymph node biopsy or lymph node resection. After correcting anemia, the left lower extremity lesion was surgically removed with a wide excision. The resection included a margin of $2-3 \mathrm{~cm}$ around the lesion and extended to the deep fascia. Following a week of closed negative pressure suction, the wound was transplanted with blade-thin skin taken from the patient's back. The skin survived well after the surgery.
The MU recurred 3 months later. The tumor and the inguinal lymph nodes were removed under general anesthesia. During the surgery, the subcutaneous and muscular layers had a fish-like appearance, and the bones were infiltrated approximately $5 \mathrm{~cm}$ below the femoral head. Osteoporosis caused the bone to look appear similar to a bean curd. The pathological diagnosis (performed by a pathologist) of the lymphoid and ulcer tissues was squamous cell carcinoma (Figs. 3 and 4). The wound was first treated with negative pressure drainage, and was grafted with skin after the granulation tissue filled the wound. The tumor did not recur during the 1 year of follow-up. 


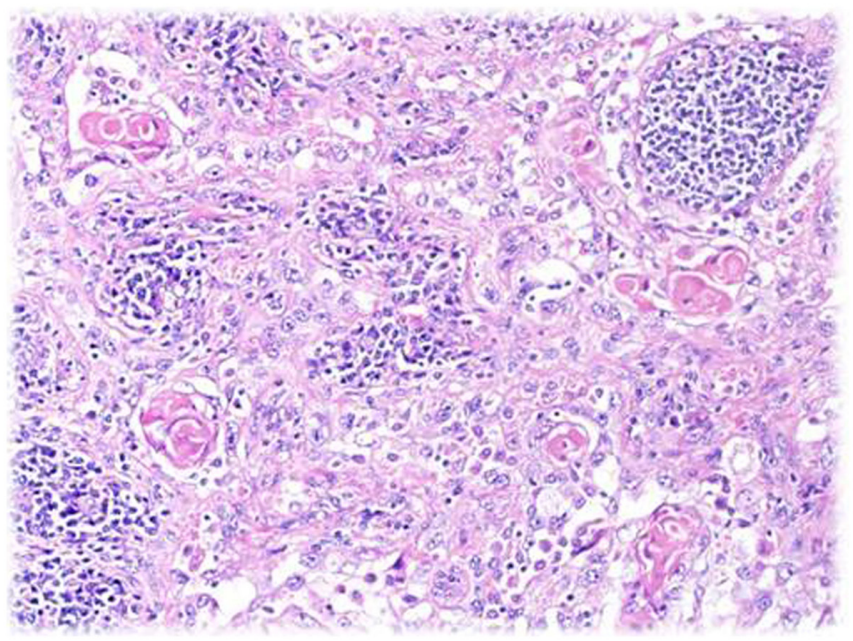

Figure 3. Pathological diagnosis of the ulcer tissue was squamous cell carcinoma (hematoxylin and eosin staining, x100 magnification).

As regards experiences and lessons learnt, he principle of MU treatment is to extensively remove the tumor. In order to achieve this, a thorough evaluation is required. This should include CT or MRI and lymph node biopsies or resection in search of metastases. The patient presented herein refused such tests and the tumor recurred with bone metastasis. A more thorough second surgical resection that included resection of the regional lymph nodes was more effective as the tumor did not recur again.

\section{Discussion}

MU is secondary to traumatized or chronically inflamed skin, particularly following burns (1-3). It is characterized as being aggressive, but has a low incidence rate (1-3). MU is often overlooked and inadequately treated (4-6). Presently, information on MU is limited. The condition remains to be defined by the medical community and guidelines for its management are required.

Definition, classification and characteristics of MU. In 1828, the French physician Jean NicholasMarjolin first described the 'warty ulcer' that occurred on scars and termed it 'cancroidal ulcer'. However, he did not know the warty ulcers he described were malignant. In 1903, Da Costa reported 2 cases of malignant ulcers on the lower extremities with a history of venous ulcers and considered them to be consistent with the ulcers described by Marjolin (7). Subsequently, the malignant ulcers occurring on chronic wounds or scars gradually began to be referred to 'Marjolin ulcers'.

In 1990, Hahn et al (8) summarized the data of 19 patients with MU and found that they were mainly 40-60-year-old males; the ulcers were primarily in the lower limbs; the primary injuries were mostly burns, followed by chronic osteomyelitis; the mean latent period was 31.5 years; the rate of metastasis at the time of diagnosis was 32\%; histopathological examination revealed that all were squamous cell carcinoma; and the average time for local recurrence was 8.8 months. Copcu et al (1) followed 264 patients with burns treated at the Izmir Ataturk Hospital from 1994 to 2001. The average follow-up time was
3.8 years. Their survey found 31 patients with MU (11.7\%) and 14 patients with benign ulcers (5.3\%). Chalya et al (9) conducted a retrospective study of patients with MU at the Bugando Medical Center, Tanzania, from 2001 to 2010. They found that MUs were not rare in their area. In 2005, Kowal-Vern and Criswell (10) found that the mean age at MU diagnosis in their study population was 50 years, and males were more commonly affected than females, with males accounting for approximately $62 \%$ of cases. Bazaliński et al (11) reviewed the history, etiopathogenesis, diagnosis and treatment of MU, and found that the early diagnosis of these wounds could reduce tissue damage and resection scope.

According to the available literature, MUs can be divided into narrowly-defined scar cancers, which occur in malignant tumors of scar ulcers $(5,12)$ caused by traumatic or chronically inflamed skin, burns in particular $(1,3)$. They are classified into acute and chronic MU based on the latency period. The latency period is $<1$ year for acute MU and $>1$ year for chronic MU (11,13-15).

The lower extremities have the highest probability of developing MU. However, MU can also occur in multiple other parts of the body, including the neck, elbow, scalp, calvarial bones, dura mater, brain, breast skin, nose and other sites (16-21). The pathological types of MU include squamous cell carcinoma, basal cell carcinoma, malignant melanoma, sarcoma, squamous basal cell carcinoma, squamous cell melanoma and other neoplasms $(10,22,23)$. Among these, squamous cell carcinoma is the most common pathological type of MU (10).

Etiology. MU causative lesions include burns (70-90\%), chronic venous ulcers, chronic injuries, scars, chronic osteomyelitis, radiation-induced wounds, diabetic foot, pressure ulcers, venous stasis, hidradenitis and others (11,24-27). There is currently no clear explanation as to the exact causes of MU. MU is considered to arise primarily from long-term chronic inflammatory stimulation that leads to mutations in focal cells. Harland et al (28) detected a homozygous deletion of the $p 53$ gene in this burn-related carcinoma. Lee et al (29) demonstrated that some burn scar-related squamous cell carcinomas had Fas gene mutations in regions important for the apoptosis function, and suggested these to be involved in the pathogenesis of the disease. Sinha et al (30) identified transcriptional changes leading to malignancy by comparing differentially expressed genes in squamous cells in squamous cell carcinoma and MU.

Diagnosis. MUs can often be accurately diagnosed based on the medical history and a physical examination. It should be suspected that the ulcer is malignant when its recovery is prolonged and the patient relapses, particularly when the secretions increase, exhibit malodor and are prone to containing blood. All patients can be further diagnosed by acquiring biopsies from the lesion. Pathological biopsies should be taken from multiple locations in the ulcer center and margin, and should go deep into the subcutaneous tissue to avoid missed diagnosis. Ultrasonography can be used as a primary modality to identify lymph node metastasis. Radiography and CT can distinguish common imaging features of MU, including bone destruction, soft tissue mass, and a periosteal reaction (31). An MRI provides excellent soft-tissue detail, such as tumor 
A

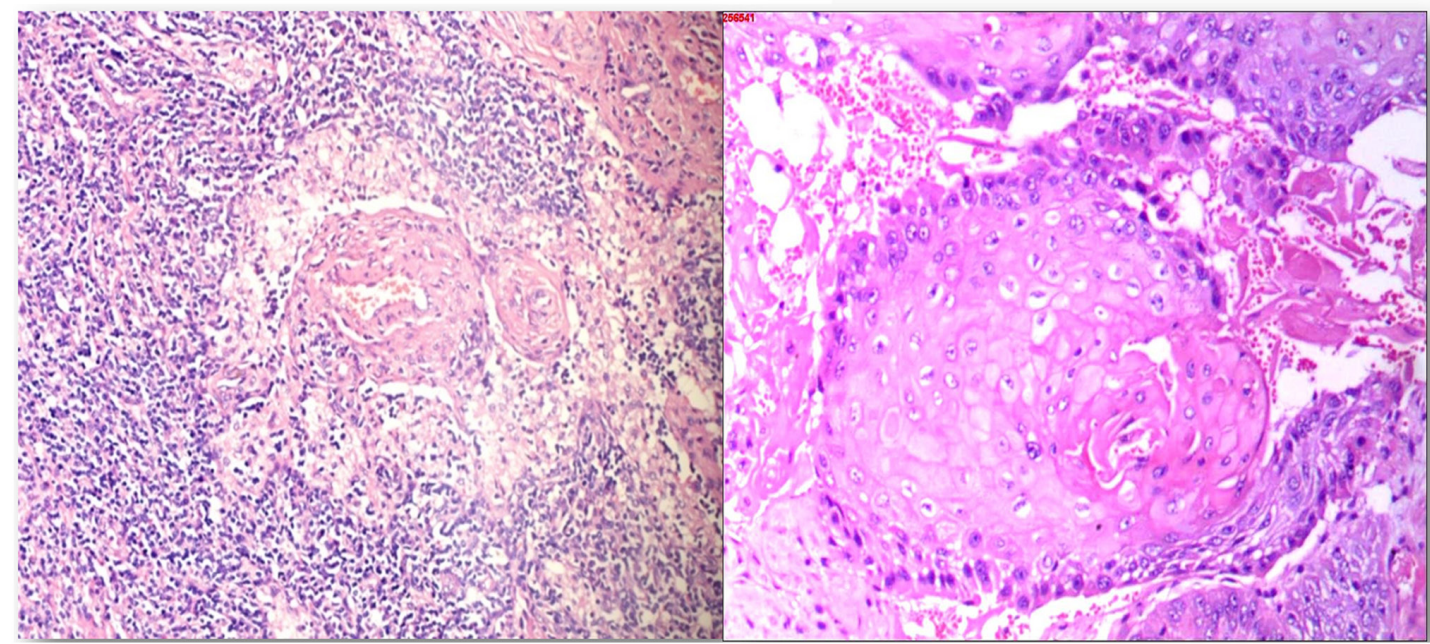

Figure 4. The inguinal lymph node histopathological diagnosis was squamous cell carcinoma. Hematoxylin and eosin staining at a magnification of (A) x100 and (B) $\mathrm{x} 200$.

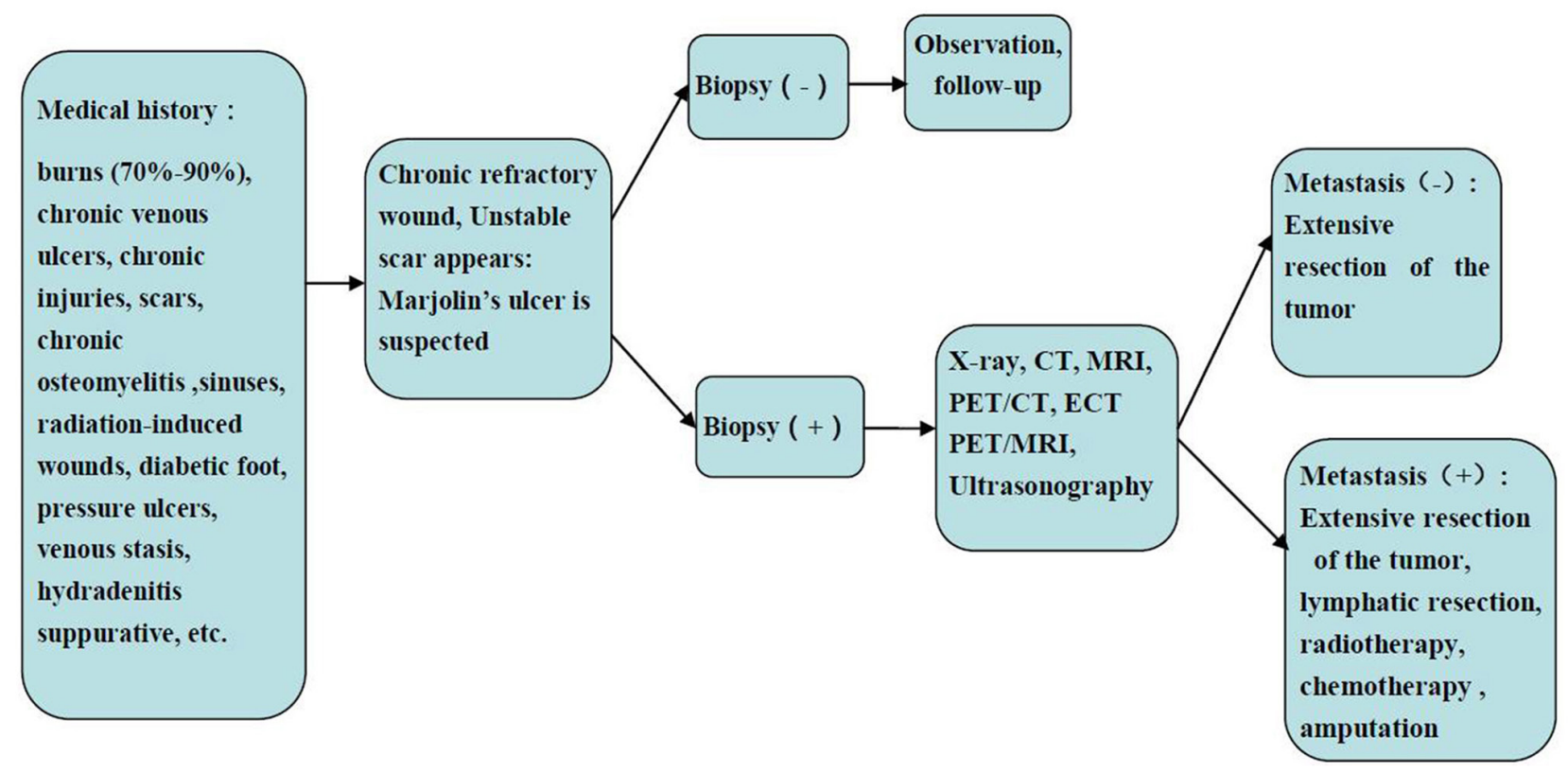

Figure 5. Flow chart of Marjolin's ulcer diagnosis and treatment.

extent, depth, margins, underlying bone cortical or marrow involvement, or the involvement of adjacent neurovascular structures (32). Positron emission tomography-CT (PET-CT) is useful in differentiating MU from benign inflammatory conditions of chronic nonhealing ulcers. It reveals a relatively good correlation with surgical or pathological results in determining the invasion depth (33). PET-MRI is feasible and performs equally well as PET-CT in the majority of cancers, with the benefit that the human body is exposed to a far lower radiation dose (34). However, there are no reports on the use of PET-MRI for the diagnosis of MU.

Treatment and prognosis. There currently no standardized treatment protocol available for MU. The extensive resection of the tumor is necessary. Bang and Woo (35) suggested that an aggressive excision and reconstruction with free tissue transfer or regional flap transposition should be adopted for adequate ablation and definitive coverage, rather than skin graft and regular surveillance. It was recommended that the smallest skin margin removed around the outer ulcer edge should be at least $2.5 \mathrm{~cm}$ (11). There is no consensus as to whether to perform sentinel node biopsy (36). If the MU is accompanied by local or distant lymph node metastasis, lymph node resection is required. Similarly, if the sentinel lymph node biopsy is positive, it is recommended to perform routine lymph node resection. For MU of the limb, amputation or hemipelvectomy (37) may be considered if the bone and joint were invaded, rendering radical resection of the lesion difficult to complete. Amputation or hemipelvectomy may also be considered if the limb function is severely impaired following radical 
resection. For the early stages of MU, an aggressive combined approach that includes extensive excision, lymphatic resection, postoperative radiotherapy and/or chemotherapy, and amputation if needed, might increase the cure rate. However, there is no consensus on advanced disease treatment, as it often produces unsuccessful results. At present, it is not recommended to routinely prescribe radiotherapy and chemotherapy to MU patients because the sensitivity of MU to these treatments is low and because of the risk of radiation-induced cell carcinogenesis. However, radiotherapy may be considered if the MU pathology is of a poorly differentiated type, the cancer has invaded the bone, there is distant metastasis, or the patient cannot tolerate or refuses to undergo surgery.

The prognosis is dictated by the time from injury to malignancy development, size, location, degree of differentiation, lymph node status, and metastasis at the time of diagnosis $(11,38,39)$.

The current literature review suggests that the diagnosis and treatment of MU should follow the flow chart illustrated in Fig. 5. It is hoped that the information provided herein will help standardize the diagnosis and treatment of MU.

In conclusion, Mus may not be an uncommon health issue with unique features. MU diagnosis and treatment guideline development would aid in the early detection and proper management of the disease, thus reducing the rates of missed diagnosis, recurrence, and mortality due to MU. However, large-scale MU clinical studies are limited; the staging criteria have not yet been established, and the choice of treatment options is mostly empirical or based on small sample observations rather than evidence-based practice. Multicenter clinical collaborative research is required to provide useful guidance for the precise diagnosis and treatment of MU.

\section{Acknowledgements}

The authors are grateful to the staff of the associated centers for providing assistance.

\section{Funding}

The present study was supported by grants from the National Natural Science Foundation of China (nos. 81171812 and 81272105), the National Basic Science and Development Program (973 Program, 2012 CB518105), the National Key Research and Development Plan of Chin (no. 2017YFC1103301), the Science and Technology Key Project of Guangdong Province (no. 2014B020212010), the Science and Technology Planning Project of Guangdong Province of China (no. 2015B020233012) and the Military Medical Innovation Special Projects (no. 18CXZ029).

\section{Availability of data and materials}

Data sharing is not applicable to this article, as no datasets were generated or analyzed during the current study.

\section{Authors' contributions}

$\mathrm{BC}$ reviewed the literature, interpreted the information, and drafted the review. JT and JPZ assisted in the preparation of the figures, complied the reference list, and revised the manuscript, and subsequently updated it as appropriate. XFX and JBT provided recommendations and assisted in the drafting and revising of the review. All authors read and approved the final manuscript.

\section{Ethics approval and consent to participate}

The present study was conducted in accordance with the 1975 Declaration of Helsinki. The study protocol was approved by the Ethics Committee of General Hospital of Southern Theater Command, People's Liberation Army. The patient provided written informed consent before investigations, screening, study and treatment.

\section{Patient consent for publication}

The patient signed an informed consent form to agree that the data can be published.

\section{Competing interests}

The authors declare that they have no competing interests.

\section{References}

1. Copcu E, Aktas A, Sişman N and Oztan Y: Thirty-one cases of Marjolin's ulcer. Clin Exp Dermatol 28: 138-141, 2003.

2. Powell HB, Googe PB and Sayed CJ: Squamous cell carcinoma arising in a chronic perineal wound in a patient with long-standing cutaneous Crohn's disease. JAAD Case Rep 4: 346-348, 2018.

3. Saaiq $M$ and Ashraf B: The menace of self-immolation plaguing low income countries. Burns 42: 472-473, 2016.

4. Pieptu D, Luchian S, Copăceanu M, Popa M, Hriscu M and Stătescu C: Marjolin's ulcer on burn scar, a curable but neglected disease. Rev Med Chir Soc Med Nat Iasi 104: 95-99, 2000 (In Romanian).

5. Fleming MD, Hunt JL, Purdue GF, Sandstad J: Marjolin's ulcer: a review and reevaluation of a difficult problem. J Burn Care Rehabil 11: 460-469, 1990

6. Kerr-Valentic MA, Samimi K, Rohlen BH, Agarwal JP and Rockwell WB: Marjolin's ulcer: Modern analysis of an ancient problem. Plast Reconstr Surg 123: 184-191, 2009.

7. Da Costa JC: III. Carcinomatous Changes in an Area of Chronic Ulceration, or Marjolin's Ulcer. Ann Surg 37: 496-502, 1903.

8. Hahn SB, Kim DJ and Jeon CH: Clinical study of Marjolin's ulcer. Yonsei Med J 31: 234-241, 1990.

9. Chalya PL, Mabula JB, Rambau P, Mchembe MD, Kahima KJ Chandika AB, Giiti G, Masalu N, Ssentongo R and Gilyoma JM: Marjolin's ulcers at a university teaching hospital in Northwestern Tanzania: A retrospective review of 56 cases. World J Surg Oncol 10: 38, 2012.

10. Kowal-Vern A and Criswell BK: Burn scar neoplasms: A literature review and statistical analysis. Burns 31: 403-413, 2005.

11. Bazaliński D, Przybek-Mita J, Barańska B and Więch P: Marjolin's ulcer in chronic wounds - review of available literature. Contemp Oncol (Pozn) 21: 197-202, 2017.

12. Metwally IH, Roshdy A, Saleh SS and Ezzat M: Epidemiology and predictors of recurrence of Marjolin's ulcer: Experience from Mansoura Universityxs. Ann R Coll Surg Engl 99: 245-249, 2017.

13. Moonsamy P, Nazarian RM, Schulz JT and Goverman J: Acute Marjolin's Ulcer Arising in a Split-Thickness Skin Graft Postburn Injury. Eplasty 16: ic31, 2016.

14. Baskara A, Sikka L, Khan F and Sapanara N: Development of a Marjolin's ulcer within 9 months in a plantar pressure ulcer. Eur J Dermatol 20: 225, 2010.

15. Mohammadi AA, Foroutan A, Mohammadi S and Anbardar MH: An odd very early marjolin's ulcer after minimal hand burn. Ann Burns Fire Disasters 30: 218-219, 2017. 
16. Simmons MA, Edwards JM and Nigam A: Marjolin's ulcer presenting in the neck. J Laryngol Otol 114: 980-982, 2000.

17. Ogawa B, Chen M, Margolis J, Schiller FJ and Schnall SB: Marjolin's ulcer arising at the elbow: A case report and literature review. Hand (N Y) 1: 89-93, 2006.

18. Gupta SK, Sandhir RK, Jaiswal AK and Kumar S: Marjolin's ulcer of the scalp invading calvarial bone, dura and brain. J Clin Neurosci 12: 693-696, 2005.

19. Gatto A, Sebastiani S, Falvo L, Giustiniani C and La Rovere C: A rare case of aggressive squamous-cell carcinoma of the breast skin: Marjolin's ulcer. A case report. Chir Ital 60: 577-582, 2008.

20. Mishra SS, Behera SK, Panigrahi S and Senapati SB: Penetrating Marjolin's ulcer of scalp with intracranial extension: A multidisciplinary experience. Asian J Neurosurg 9: 240, 2014.

21. Bhasme V, Agrawal M, Poonia NC, Bagaria H and Chakotiya P: Post-burn spontaneous brain fungation caused by infiltrating Marjolin's ulcer of scalp. Asian J Neurosurg 12: 256-258, 2017.

22. Koh SH, Oh SJ, Chun H and Kim SG: Pseudoangiosarcomatous squamous cell carcinoma developing on a burn scar: A case report and review of the literature. Burns 40: e47-e52, 2014.

23. Noori VJ, Trehan K, Savetamal A and Carter DW: New onset squamous cell carcinoma in previous split-thickness skin graft donor site. Int J Surg 52: 16-19, 2018.

24. Nthumba PM: Marjolin's ulcers: Theories, prognostic factors and their peculiarities in spina bifida patients. World J Surg Oncol 8: $108,2010$.

25. Eliassen A, Vandy F, McHugh J and Henke PK: Marjolin's ulcer in a patient with chronic venous stasis. Ann Vasc Surg 27: 1182. e5-1182.e8, 2013.

26. Yon JR, Son JD, Fredericks C, Morton M, Kingsley S, Gupta S, Poulakidas S and Bokhari F: Marjolin's Ulcer in Chronic Hidradenitis Suppurativa: A Rare Complication of an Often Neglected Disease. J Burn Care Res 38: 121-124, 2017.

27. Garcia-Marín JA, de Alcala Martinez-Gomez D, Campillo-Soto A and Aguayo-Albasini JL: Marjolin's ulcer. A 10 year experience in a diabetic foot unit. Cir Cir 84: 340-343, 2016 (In Spanish).

28. Harland DL, Robinson WA and Franklin WA: Deletion of the p53 gene in a patient with aggressive burn scar carcinoma. J Trauma 42: 104-107, 1997.

29. Lee SH, Shin MS, Kim HS, Park WS, Kim SY, Jang JJ, Rhim KJ, Jang J, Lee HK, Park JY, et al: Somatic mutations of Fas (Apo-1/CD95) gene in cutaneous squamous cell carcinoma arising from a burn scar. J Invest Dermatol 114: 122-126, 2000.
30. Sinha S, Su S, Workentine M, Agabalyan N, Cheng M, Gabriel V and Biernaskie J: Transcriptional analysis reveals evidence of chronically impeded ECM turnover and epithelium to mesenchyme transition in scar tissue giving rise to Marjolin's ulcer. J Burn Care Res 38: e14-e22, 2017.

31. Mohamed S, Abdullah B, Singh DA and Heng KS: CT appearances of Marjolin's ulcer in the left gluteal region of a young man. Biomed Imaging Interv J 2: e26, 2006.

32. Sawhney S, Jain R, Kakaria A and Chopra P: Marjolin's Ulcer: Radiographic and magnetic resonance appearances in two cases. Sultan Qaboos Univ Med J 9: 162-166, 2009.

33. Ko Y, Han YM, Hwang HS, Kang IW, Hwang DH, Lee ES and Lee GK: Role of ${ }^{18} \mathrm{~F}-\mathrm{FDG}$ PET/CT in the diagnosis of clinically suspected Marjolin ulcer. AJR Am J Roentgenol 199: 1375-1379, 2012.

34. Lee MS, Cho JY, Kim SY, Cheon GJ, Moon MH, Oh S, Lee J, Lee S, Woo S and Kim SH: Diagnostic value of integrated PET/MRI for detection and localization of prostate cancer: Comparative study of multiparametric MRI and PET/CT. J Magn Reson Imaging 45: 597-609, 2017.

35. Bang CY and Woo SH: The Fate of Chronic Burn Wounds Suspected as Marjolin's Ulcers. J Burn Care Res 39: 148-153, 2018.

36. Motamedolshariati M, Rezaei E, Beiraghi-Toosi A, Jahani A, Tayyebi Meibodi N, Fattahi A and Sadeghi R: Sentinel node mapping in Marjolin's ulcers: Is it feasible? Wounds 27: 54-62, 2015.

37. Carlesimo B, Monarca C, Rizzo MI, Tariciotti F and Staccioli S: Hemipelvectomy and reconstruction in a patient with advanced Marjolin's ulcer: A case report. Int J Low Extrem Wounds 8: 162-164, 2009.

38. Aydoğdu E, Yildirim S and Aköz T: Is surgery an effective and adequate treatment in advanced Marjolin's ulcer? Burns 31: 421-431, 2005.

39. Pekarek B, Buck S and Osher L: A Comprehensive Review on Marjolin's Ulcers: Diagnosis and Treatment. J Am Col Certif Wound Spec 3: 60-64, 2011.

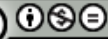

This work is licensed under a Creative Commons Attribution-NonCommercial-NoDerivatives 4.0 International (CC BY-NC-ND 4.0) License. 